Bull. Austral. Math. Soc.

$54 \mathrm{C} 05,54 \mathrm{C} 08,54 \mathrm{C} 10,54 \mathrm{D} 10,54 \mathrm{D} 05$

VOL. 41 (1990) [57-74]

\title{
A UNIFIED APPROACH TO CONTINUOUS AND CERTAIN NON-CONTINUOUS FUNCTIONS II
}

\author{
J.K. KOHLI
}

\begin{abstract}
A unified theory of continuous and certain non-continuous functions, initiated in an earlier paper, is further elaborated. The proposed theory provides a common platform for dealing simultaneously with continuous functions and a host of non-continuous functions including lower (upper) semicontinuous functions, almost continuous functions, weakly continuous functions (encountered in functional analysis), $c$-continuous functions, $\delta$-continuous functions, semiconnected functions, $H$-continuous functions s-continuous functions, $\varepsilon$-continuous functions of Klee and several other variants of continuity.
\end{abstract}

\section{INTRODUCTION.}

In [19] we developed a unified and coherent theory of continuous and certain noncontinuous functions and introduced the notions of $P$-continuous functions and semi$P$-functions (or $P$-proper maps) as unifying tools. It turns out that the theory of $P$-continuous functions encompasses in one the theories of continuous functions, upper (lower) semicontinuous functions, $c$-continuous functions [12], almost continuous functions $[51], c^{*}$-continuous functions [46], s-continuous functions [20], $H$-continuous functions $[30], \ell$-continuous functions $[23], \tau_{i}$-continuous functions $[1], \varepsilon$-continuous functions $([17,18])$, and several other generalisations of continuity. In the same vein the theory of $P$-proper maps (= semi $P$-functions) provides a unified framework for dealing with continuous functions, $\delta$-continuous functions [44], semiconnected functions $([16,28]), z$-continuous functions [53], strongly c-continuous functions [10], strongly $\ell$-continuous functions $[11], R$-maps $([4,45])$, and other similar variants of continuity.

Certain of the variants of continuity mentioned above arise in topological applications, while others have been studied because of their intrinsic interest and yet others seem natural in connection with the mathematical modelling of certain physical problems (see for example [18]). The notions of $P$-continuous functions and $P$-proper maps also arise naturally in many topologico-analytic situations, where there is an interplay between two or more topologies on the same underlying set. For example, in locally convex linear topological spaces the interrelations among strong topology, weak topology, Mackey topology and other topologies lead to considerations which fall under the

Received 26 January 1989

Copyright Clearance Centre, Inc. Serial-fee code: 0004-9729/90 \$A2.00+0.00. 
purview of $\boldsymbol{P}$-continuous functions and $P$-proper maps. Similarly, on the space of operators on a Hilbert space, interaction among the norm topology, strong operator topology and weak operator topology leads to analogous situations. In this paper we continue our study in the style of [19] and elaborate further on the theory of $P$-continuous functions and $P$-proper maps. Applications of $P$-continuous functions and $P$-proper maps to functional analysis will be published elsewhere. The accompanying table shows the variants of continuity we shall consider in this paper.

REMARK. In addition to those in the table, notions of (weakly) $\Gamma$-continuous functions due to Wagner [61], $\gamma$-continuous functions introduced by Commaroto and Faro [6], $W C$-continuous studied by Commarato and Noiri [7], and weakly continuous functions studied in functional analysis also fit nicely into the present framework.

Section 2 is devoted to preliminaries and a brief review of the unified framework is given in Section 3. Basic properties of $P$-continuous functions and $P$-proper maps are studied in Section 4. In Section 5, we obtain several sufficient conditions for the continuity of $P$-continuous functions. The concept of $P$-Hausdorff space is introduced in Section 6. The notion of a $P$-Hausdorff space represents an abstraction of the concepts of a Hausdorff space, functionally Hausdorff space, ultra Hausdorff space, (countably) compact Hausdorff space and many other topological invariants.

\section{BASIC DEFINITIONS AND PRELIMINARIES}

Let $X$ be a topological space. A subset $A$ of $X$ is said to be regularly closed if it is the closure of its interior. A $\delta$-closed set $[60]$ is the intersection of any collection of regularly closed sets and a $\pi$-set $[63]$ is the intersection of finitely many regularly closed sets. A $z$-closed set is the intersection of any collection of zero sets. The complement of a regularly closed set is called regularly open. A subset $A$ of $X$ is said to be strongly regularly open [59] if $A=$ int $\operatorname{ker} \mathrm{Cl} A$, where $\operatorname{ker} B$ denotes the intersection of all open sets containing $B$. The complement of a strongly regular open set is called strongly regular closed. A subset $F$ of $X$ is said to be a point closure [24] if it is the closure of a singleton.

A subset $A$ of a space $X$ with topology $\tau$ is called quasi $H$-closed relative to $X$ [47] if each $\tau$-open family which covers $A$ has a finite subfamily whose union is $\tau$-dense in $A$. A subset $S$ of $X$ is said to be $N$-closed [5] if for any open cover $\mathcal{U}$ of $S$ there is a finite subcollection $\mathcal{V}$ of $\mathcal{U}$ such that $S \subseteq \bigcup\{$ int $\mathrm{Cl} V \mid V \in \mathcal{V}\}$.

A family $\mathcal{F}$ of closed sets in $X$ is called a strongly closed $G_{6}$-family [3] if each $F \in \mathcal{F}$ is the countable intersection $F=\bigcap\left\{X-F_{i} \mid F_{i} \in \mathcal{F}\right\}$; the members of any such family $\mathcal{F}$ are called strongly closed $G_{\delta}-s e t s[3]$. It turns out that strongly closed $G_{\delta}$-sets are precisely the $D$-closed sets [13]. 


\section{A UNIFIED FRAMEWORK}

Throughout the paper $P$ will denote a topological property enjoyed by certain subsets of a topological space. In the original formulation [19] property $P$ is not necessarily required to be a topological invariant. We adopt this restriction for similicity; it essentially covers all the variants of continuity discussed in this paper except the $\varepsilon$ continuity of Klee [17]. The properties with which we shall be dealing in this paper are quite diverse and include among others, the property of being a zero set, or a $G_{\delta^{-}}$ set, being a (regularly) closed set, being a point closure, being a connected set or a compact set, being a strongly regular closed set et cetera; a complete list is given in the accompanying table.

Definition 3.1. ([19, 24]). Let $X$ be a topological space and let $A \subseteq X$. We say that

(i) $A$ is a $P$-set if $A$ possesses property $P$;

(ii) $A$ has $P$-complement if $X-A$ possesses property $P$.

DEFINITION 3.2. A property $P$ is said to be finitely additive (multiplicative) if the finite union (intersection) of $P$-sets is a $P$-set.

Definition 3.3. [19] Let $f: X \rightarrow Y$ be a function from a topological space $X$ into a topological space $Y$. Then $f$ is called

(1) $P$-continuous if for each $x \in X$ and each open set $V$ containing $f(x)$ and having $P$-complement there is an open set $U$ containing $x$ such that $f(U) \subset V$;

(2) a $P$-proper map if for each closed $P$-set $K \subseteq Y, f^{-1}(K)$ is a closed $P$-set in $X$.

As was remarked in the introduction, the notions of $\boldsymbol{P}$-continuous functions and $P$-proper maps represent a simultaneous abstraction of the concepts of continuous functions and a host of other non-continuous functions. We refer the reader to the table for the type of $P$-continuous function and $P$-proper map corresponding to a particular property $P$. Referencs are quoted as an aid to the literature and an attempt has been made to include the reference in which a concept or notion appears for the first time. However, no claim is made to completeness or originality of the source.

Remark 3.1. If $P_{1}$ and $P_{2}$ denote two properties such that $P_{1}$ implies $P_{2}$, then every $P_{2}$-continuous function is a $P_{1}$-continuous function while the reverse implication does not hold in general. In contrast to this the classes of $P_{1}$-proper maps and $P_{2}$-proper maps may not be even comparable in general. For example, if $P_{1}=$ regularly closed (respectively, $\delta$-closed) set and $P_{2}=$ closed set, then a $P_{1}$-proper map $\equiv$ an $R$-map 
[4] (respectively, $\delta$-continuous function [44]) and a $P_{2}$-proper map $\equiv$ a continuous function.

THEOREM 3.1. [19] Let $f: X \rightarrow Y$ be a function from a topological space $X$ into a topological space $Y$. The following statements are equivalent:

(a) $f$ is $P$-continuous;

(b) if $V$ is an open subset of $Y$ having $P$-complement, then $f^{-1}(V)$ is an open subset of $X$;

(c) for each closed $P$-set $K \subseteq Y, f^{-1}(K)$ is closed in $X$.

\section{Properties of $P$-continuous functions and $P$-Proper maps}

In this section we continue to investigate the properties of $P$-continuous functions and $P$-proper maps in the same spirit as in [19]. It will become clear in the sequel that the results obtained in the process embody in themselves so much information that besides improving and unifying known results, they often suggest new results.

TheOREM 4.1. Let $P$ denote a topological property such that a product space has $P$ if and only if each co-ordinate space has $P$. For each $\alpha \in \Lambda$, let $f_{\alpha}: X_{\alpha} \rightarrow Y_{\alpha}$ be a function such that $Y_{\alpha}$ possesses property $P$. Let $f: \prod X_{\alpha} \rightarrow \prod Y_{\alpha}$ be defined by $f\left(\left(x_{\alpha}\right)\right)=\left(f_{\alpha}\left(x_{\alpha}\right)\right)$ for each $\left(x_{\alpha}\right)$ in $\prod X_{\alpha}$. If $f$ is a $P$-proper function, then each $f_{\alpha}$ is a $P$-proper function and each $X_{\alpha}$ has property $P$.

Proof: For each $\alpha \in \Lambda$, let $p_{\alpha}: \prod X_{\alpha} \rightarrow X_{\alpha}$ and $q_{\alpha}: \prod Y_{\alpha} \rightarrow Y_{\alpha}$ be projections. Then in view of the definition of $f$, it follows that $q_{\alpha} \circ f=f_{\alpha} \circ p_{\alpha}$, for each $\alpha \in \Lambda$. Let $K \subseteq Y_{\alpha}$ be any closed $P$-set. Now, since each $Y_{\alpha}$ possesses property $P$ and since $P$ is productive, $q_{\alpha}^{-1}(K)=\left(\prod_{\beta \neq \alpha} Y_{\beta}\right) \times K$ is a closed $P$-set in $\prod Y_{\alpha}$. Again, since $f$ is a $P$-proper function, $f^{-1}\left(q_{\alpha}^{-1}(K)\right)$ is a closed $P$-set in $\prod X_{\alpha}$. But

$$
\begin{aligned}
& f^{-1}\left(q_{\alpha}^{-1}(K)\right)=\left(q_{\alpha} \circ f\right)^{-1}(K)=\left(f_{\alpha} \circ p_{\alpha}\right)^{-1}(K)=p_{\alpha}^{-1}\left(f_{\alpha}^{-1}(K)\right) \\
&=\left(\prod_{\beta \neq \alpha} X_{\beta}\right) \times f_{\alpha}^{-1}(K) . \\
& \ddots
\end{aligned}
$$

By the hypothesis on the property $P$, it is immediate that $f_{\alpha}^{-1}(K)$ is a closed $P$-set in $X_{\alpha}$ and so $f_{\alpha}$ is a $P$-proper function. Since each $Y_{\alpha}$ enjoys property, it follows that each $X_{\alpha}$ also enjoys $P$.

REMARK 4.1. If $P$ denotes connectedness in the above theorem, then we obtain the latter part of Theorem 2.2 of [21] pertaining to semiconnected functions. Similarly, the substitution $P=\delta$-closed set in Theorem 4.1 yields a result of Mathur [37] pertaining 
to $\delta$-continuous functions and with $P=$ zero set it gives a corresponding version for $z$-continuous function.

ThEOREM 4.2. Let $P$ denote a property which is productive. For each $\alpha \in \Lambda$, let $f_{\alpha}: X_{\alpha} \rightarrow Y_{\alpha}$ be a function such that each point of $Y_{\alpha}$ is contained in a closed $P$-subset of $Y_{\alpha}$. Suppose $f: \prod X_{\alpha} \rightarrow \prod Y_{\alpha}$, defined by $f\left(x_{\alpha}\right)=\left(f_{\alpha}\left(x_{\alpha}\right)\right)$ for each $\left(x_{\alpha}\right)$ in $\prod_{\alpha}$, is $P$-continuous. Then each $f_{\alpha}$ is $P$-continuous.

Proof: Let $F_{\beta}$ be a closed $P$-subset of $Y_{\beta}$ and let $\left(y_{\alpha}^{0}\right)$ be a point in the image of $f$. By hypothesis on the spaces $Y_{\alpha}$, for each $\alpha$, there is a closed $P$-subset $K_{\alpha}$ of $Y_{\alpha}$ containing $y_{\alpha}^{0}$. In view of the productivity of the property $P$, it follows that $F_{\beta} \times\left(\prod_{\alpha \neq \beta} K_{\alpha}\right)$ is a closed $P$-subset of $\prod Y_{\alpha}$. Since $f$ is $P$-continuous, by Theorem 3.1, we infer that $f^{-1}\left(F_{\beta} \times\left(\prod_{\alpha \neq \beta} K_{\alpha}\right)\right)$ is closed in $\Pi X_{\alpha}$. But $f^{-1}\left(F_{\beta} \times\left(\prod_{\alpha \neq \beta} K_{\alpha}\right)\right)=f_{\beta}^{-1}\left(F_{\beta}\right) \times\left(\prod_{\alpha \neq \beta} f_{\alpha}^{-1}\left(K_{\alpha}\right)\right)$ and so $f^{-1}\left(F_{\beta}\right)$ is closed in $X_{\beta}$ and thus $f_{\beta}$ is $P$-continuous.

REMARK 4.2. Reading from the table, the above theorem contains several results in the literature. For example, with $P=$ conpactness it gives an assertion which generalises Theorem 2.3 of Long and Herrington [33] pertaining to $c$-continuous functions and with $P=$ regularly closed it yields Theorem 1 of Long and Herrington [32] (this is also the necessity part of Theorem 1 of Noiri [40]) concerning almost continuous functions. Similarly, with $P=$ quasi $H$-closed it yields a version which generalises Theorem 4.3 of Noiri [42] pertaining to $H$-continuous functions.

Theorem 4.3. Let $f: X \rightarrow \prod X_{\alpha}$ be a $P$-continuous function into a product space such that each $X_{\alpha}$ enjoys property $P$, and suppose that $P$ is productive. Then each $p_{\alpha} \circ f$ is $P$-continuous.

Proof: Let $F_{\beta}$ be a closed $P$-subset of $X_{\beta}$. Then

$$
\left(p_{\beta} \circ f\right)^{-1}\left(F_{\beta}\right)=f^{-1}\left(p_{\beta}^{-1}\left(F_{\beta}\right)\right)=f^{-1}\left(F_{\beta} \times\left(\prod_{\alpha \neq \beta} X_{\alpha}\right)\right)
$$

and in view of the productivity of $P$ it follows that, $F_{\beta} \times\left(\prod_{\alpha \neq \beta} X_{\alpha}\right)$ is a closed $P$-set in $\prod X_{\alpha}$. Now, since $f$ is $P$-continuous, the result is immediate in view of Theorem 3.1. 
Remark 4.3. The substitution $P=$ connectedness in Theorem 4.3 gives [21, Theorem 2.3] pertaining to $s$-continuous functions and with $P=$ regularly closed set it yields Theorem 2.11 of Singal and Singal [51] concerning almost continuous functions (see also Long and Herrington [32, Theorem 3]). Moreover, the substitution $P=$ quasi $H$-closed in Theorem 4.3 yields an assertion which shows that " $H$-closed" in Theorem 4.7 of Noiri [42] can be weakened to "quasi $H$-closed".

Theorem 4.4. Let $f: X \rightarrow Y$ be a $P$-continuous function such that for each relatively closed $P_{\text {-set }} A \subseteq f(X), \mathrm{Cl}_{Y} A$ is a $P$-set. Then $f: X \rightarrow f(X)$ is $P$ continuous.

Proof: Let $K$ be a relatively closed $P$-subset of $f(X)$. Then $\mathrm{Cl}_{Y} K$ is a closed $P$-subset of $Y$. Since $f$ is $P$-continuous, by Theorem $3.1, f^{-1}\left(\mathrm{Cl}_{Y} K\right)$ is closed in $X$, and the conclusion is immediate in view of the fact that $f^{-1}(K)=f^{-1}\left(\mathrm{Cl}_{Y} K\right)$.

REMARK 4.4. Since a compact set in a Hausdorff space is closed, the substitution $P=$ compactness in Theorem 4.1 yields an assertion which includes Theorems 2.13 and 2.14 of Long and Herrington [33] pertaining to $c$-continuous functions. Similarly, the substitution $P=$ countable compactness gives a version which includes Theorems 3.4 and 3.5 of Long and Herrington [33] concerning $c^{*}$-continuous functions.

REMARK 4.5. The function $f: X \rightarrow Y$ in [33, Example 2.12] is $c^{*}$-continuous but $f: X \rightarrow f(X)$ is not even $c$-continuous. Thus the hypothesis on $Y$ in Theorem 4.4 cannot be omitted.

TheOREM 4.5. Let $f: X \rightarrow Y$ be either an open or closed surjection, and let $g: Y \rightarrow Z$ be any function such that $g \circ f$ is $P$-continuous. Then $g$ is $P$-continuous.

Proof: Suppose $f$ is open (respectively, closed), and let $V \subseteq Z$ be an open set having $P$-complement (respectively, $V \subseteq Z$ be a closed $P$-set). Since $g \circ f$ is $P$-continuous, by Theorem $3.1(g \circ f)^{-1}(V)=f^{-1}\left(g^{-1}(V)\right)$ is an open set (respectively, a closed set). Since $f$ is an open surjection (respectively, a closed surjection), $f\left(f^{-1}\left(g^{-1}(V)\right)\right)=g^{-1}(V)$ is an open set (respectively, a closed set) and thus $g$ is $P$-continuous.

REMARK 4.6. The substitution $P=$ connectedness in Theorem 4.6 yields $[21$, Theorem 2.5] on $s$-continuous functions and with $P=$ regularly closed set we get an assertion which is a significant improvement of Theorem 2.5 of Singal and Singal [51] pertaining to almost continuous functions.

REMARK 4.7. The hypothesis of being either open or closed in Theorem 4.5 cannot be omitted (see [21, Remark 2.2]).

THEOREM 4.6. Let $g: Y \rightarrow Z$ be a function which maps $P$-sets in $Y$ to $P$-sets 
in $Z$. Let $f: X \rightarrow Y$ be a function such that $g \circ f$ is $P$-continuous. If $g$ is an open bijection (or a closed injection), then $f$ is $P$-continuous.

Proof: Case $I . g$ is an open bijection. Let $V$ be an open set in $Y$ having $P$ complement. Since $g$ preserves $P$-sets and since $g$ is an open bijection, $g(Y-V)=$ $Z-g(V)$ and so $g(V)$ is an open set in $Z$ having $P$-complement. Again, since $g \circ$ $f$ is $P$-continuous, in view of Theorem 3.1 and the injectivity of $g$, it follows that $(g \circ f)^{-1} \cdot(g(V))=f^{-1}\left(g^{-1}(g(V))\right)=f^{-1}(V)$ is an open set in $X$ and hence $f$ is $P$ continuous.

Case II. $g$ is a closed injection. Let $F$ be a closed $P$-set in $Y$. Then $g(F)$ is a closed $P$-set in $Z$, since $g$ preserves $P$-sets. Again, since $g \circ f$ is $P$ continuous, by Theorem 3.1 and injectivity of $g$ if follows that $(g \circ f)^{-1}(g(F))=f^{-1}\left(g^{-1}(g(F))\right)=f^{-1}(F)$ is a closed set in $X$ and thus $f$ is $P$-continuous.

Remark 4.8. The substitution $P=$ connectedness in Theorem 4.6 yields an assertion which includes Theorem 2.6 of $[21]$ pertaining to $s$-continuous functions. Similarly, with $P=$ closed set it shows that if $g$ is a closed injection and $g \circ f$ is continuous, then $f$ is continuous.

DEFINITION 4.1. [34]. A function $f: X \rightarrow Y$ is said to have strongly closed graph in the case that for each $(x, y) \notin G(f)$ there exist open sets $U$ and $V$ containing $x$ and $y$, respectively, such that $U \times \bar{V}$ is disjoint from $G(f)$.

Theorem 4.7. Let $f: X \rightarrow Y$ be a $P$-continuous function into a Hausdorff space $Y$ such that each point in $Y$ has a neighbourhood whose closure is a $P$-set. If $P$ is a regularly closed hereditary property, then $f$ has strongly closed graph.

Proof: Let $(x, y) \notin G(f)$. Then $f(x) \neq y$ and so in view of the Hausdorffness of $Y$ there is an open set $V$ containing $y$ such that $f(x) \notin \bar{V}$. By the hypothesis on $Y$ there exists an open set $W$ in $Y$ containing $y$ such that $\bar{W}$ is a $P$-set. Then $V \cap W$ is an open set containing $y$ such that $\overline{V \cap W} \subseteq \bar{W}$. Since the closure of an open set is regularly closed and since $P$ is a regularly closed hereditary property, $\bar{V} \cap \bar{W}$ is a $P$-set which does not contain $f(x)$. Therefore, $Y-\overline{V \cap W}$ is an open set containing $f(x)$ and having $P$-complement and so in view of the $P$-continuity of $f$, there is an open set $U$ in $X$ containing $x$ such that $f(U) \subseteq Y-\overline{V \cap W}$. Consequently, $U \times \overline{V \cap W}$ contains no point of $G(f)$, and hence $G(f)$ is strongly closed.

REMARK 4.9. Reading from the table, Theorem 4.7 contains several results in the literature. Since a regularly closed subset of a $H$-closed space is $H$-closed, the substitution $P=$ quasi $H$-closed (respectively, $P=$ countable compactness, respectively, $P=$ regularly closed compact set) in Theorem 4.7 yields an assertion which generalises Theorem 8 of Long and Hamlett [30] (respectively, [33, Theorem 3.2] of Long and Herrington, 
respectively [43, Theorem 3.4] of Noiri). Similarly, with $P=$ regularly closed it shows that an almost continuous function into a Hausdorff space has strongly closed graph, a result due to Long and Herrington [32, Theorem 1] (also see Noiri [41, Theorem 1]).

Theorem 4.8. Let $P$ denote a property enjoyed by every clopen set. Then every $P$-continuous image of a connected space is connected.

Proof: Let $f: X \rightarrow Y$ be a $P$-continuous surjection from a connected space $X$ onto a space $Y$. Suppose $Y$ is not connected and let $Y=A \cup B$ be a partition of $Y$. Then $A$ and $B$ are nonempty complementary clopen sets and hence $P$-subsets of $Y$. In view of the $P$-continuity of $f$, it follows that $f^{-1}(A)$ and $f^{-1}(B)$ are open sets in $X$. Again, since $f^{-1}(A)$ and $f^{-1}(B)$ are disjoint, $X=f^{-1}(A) \cup f^{-1}(B)$ constitutes a partition of $X$. This contradiction to the fact that $X$ is connected shows that $Y$ is connected.

REMARK 4.10. Since every clopen set is regularly closed, with $P=$ regularly closed Theorem 4.8 yields Theorem 4 of Long and Carnahan [29] pertaining to almost continuous functions. The substitution $P=$ clopen set in Theorem 4.8 shows that a mildly continuous image of a connected space is connected [54]. Similarly, with $P=$ zero set we obtain that the $z$-continuous image of a connected space is connected [53].

\section{Continuity of P-continuous functions}

In [19], we obtained several sufficient conditions on the domain and/or range implying continuity of $P$-continuous functions. In this section we offer a few more sufficient conditions, in the spirit of [19], which in their turn yield sufficient conditions for continuity of a host of not necessarily continuous functions. The results obtained in the process improve and unify scores of known results on various non-continuous functions, and often suggest new results.

Definition 5.1. [8]. A subset $A$ of a topological space $X$ is said to be sequentially open if whenever a sequence in $X$ converges to a point in $A$, it is eventually in $A$. A topological space $X$ is said to be sequential if every sequentially open set in $X$ is open.

Theorem 5.1. Let $f: X \rightarrow Y$ be a $P$-continuous function from a sequential space $X$ into a countably compact Hausdorff space $Y$ such that $Y$ possesses a base of closed $P$-neighbourhoods. Then $f$ is continuous.

Proof: Suppose $f$ is not continuous. Then there is an open set $Y \subseteq Y$ such that $f^{-1}(V)$ is not open in $X$ and so in view of the fact that $X$ is a sequential space, $f^{-1}(V)$ is not sequentially open. Hence there is a sequence $\left\{x_{n}\right\}$ in $X-f^{-1}(V)$ which converges to a point $x \in f^{-1}(V)$, and the sequence $\left\{f\left(x_{n}\right)\right\}$ does not converge to $f(x)$. By the countable compactness of $Y$, the sequence $\left\{f\left(x_{n}\right)\right\}$ clusters at a point $y \in Y-V$. By 
the Hausdorffness of $Y$ there are disjoint open sets $V_{1}$ and $V_{2}$ containing $f(x)$ and $y$, respectively, and such that $V_{1} \subseteq V$. Also there is a closed $P$-neighbourhood $W=\bar{W}$ of $y$ such that $W \subseteq V_{2}$. Thus $W$ is a closed $P$-set such that $f^{-1}(W)$ is not closed because it does not contain its limit point $x$. This contradiction to Theorem 3.1 shows that $f$ is continuous.

REMARK 5.1. Since a first countable space is sequential, Theorem 5.1 includes Theorem 4.5 of [19]. Moreover, it contains several results in the literature. For example, with $P=$ compactness it yields Theorem 2.11 of $[23]$ pertaining to $c$-continuous functions and hence includes Theorem 12 of Long and Hendrix [31]. Similarly, with $P=$ connectedness it gives Theorem 3.1 of $[22]$ pertaining to $s$-continuous functions and thus includes Theorem 2.1 of [21].

THEOREM 5.2. Suppose $P$ is a property which is closed hereditary and let $f$ : $X \rightarrow Y$ be a $P$-continuous function such that $f(X)$ is contained in a closed $P$-subset of $Y$. Then $f$ is continuous.

Proof: Let $F$ be a closed $P$-subset of $Y$ containing $f(X)$ and let $V$ be any open subset of $Y$. Since $P$ is closed hereditary, $V \cup(Y-F)$ is an open subset of $Y$ having $P$-complement. Since $f$ is $P$-continuous by Theorem $3.1 f^{-1}(V \cup(Y-F))$ is open in $X$ and the proof is complete in view of the fact that $f^{-1}(V)=f^{-1}(V \cup(Y-F))$.

REMARK 5.2. Using the table, Theorem 5.2 contains several known results in the literature. For example, with $P=$ compactness it gives Theorem 5 of Gentry and Hoyle [12] pertaining to $c$-continuous functions and with $P=$ Lindelöfness it yields Theorem 2.9 of [23] corresponding to $\ell$-continuous functions. Similarly, for $P=$ countable compactness it gives a sufficient condition for a $c^{*}$-continuous function to be continuous.

Theorem 5.3. Let $f: X \rightarrow Y$ be a $P$-continuous connected function from a locally connected space $X$ into a metric space $(Y, d)$ in which every closed and bounded set is a $P$-set. Then $f$ is continuous.

Proof: Let $x \in X$ and let $N_{e}(f(x))$ be a basic open set in $Y$ containing $f(x)$. Let $V=Y-\overline{N_{2 \varepsilon}(f(x))}$. Then $N_{\varepsilon}(f(x)) \cup V$ is an open subset of $Y$ containing $f(x)$ whose complement is a closed and bounded set in $Y$ and hence a $P$-set. Since $f$ is $P$-continuous, there is an open set $W$ containing $x$ such that $f(W) \subseteq N_{e}(f(x)) \cup V$. Since $X$ is locally connected, we may assume that $W$ is connected and consequently, $f(W)$ is connected, $f$ being a connected function. Again, since $N_{\varepsilon}(f(x))$ and $V$ are disjoint open sets, and since $f(x) \in f(W) \cap N_{\varepsilon}(f(x)), f(W) \subseteq N_{\varepsilon}(f(x))$, and so $f$ is continuous.

REMarK 5.3. According to the table, the above theorem includes several results in the literature. For example, with $P=$ compactness it yields Theorem 8 of Gentry and 
Hoyle [12] concerning $c$-continuous functions and for $P=$ the Lindelof property it gives Theorem 2.10 of $[\mathbf{2 3}]$ pertaining to $\ell$-continuous functions.

Theorem 5.4. Suppose $P$ is closed hereditary and let $f: X \rightarrow Y$ be a $P$ continuous function from a space $X$ into a metric space $Y$ such that every closed and bounded subset of $Y$ is a $P$-set. If $f$ is locally bounded, then $f$ is continuous.

Proof: Let $x$ be any point in $X$. Since $f$ is locally bounded, there is an open set $U$ containing $x$ such that $f(U)$ is bounded and so $f(U)$ is contained in a closed and bounded set $K \subseteq Y$, which in view of the hypothesis on $Y$ is a $P$-set. Again, since $f$ is $P$-continuous, by [19, Theorem 3.6] $f \mid U: U \rightarrow Y$ is $P$-continuous. Thus, the restriction $f \mid U: U \rightarrow Y$ satisfies the hypotheses of Theorem 5.2 and hence it is continuous. Consequently, $f$ is continuous on $X$.

REMARK 5.4. The substitution $P=$ compactness in the above theorem yields Theorem 2.4 of Long and Herrington [33] pertaining to c-continuous functions.

\section{P-HAUSDORFF SPACES}

In this section we introduce the notion of a $P$-Hausdorff space which represents a simultaneous abstraction of the concepts of a Hausdorff space, functionally Hausdorff space, ultra Hausdorff space [48], (countably) compact Hausdorff space, Lindelöf Hausdorff space and many other topological invariants.

Definition 6.1. ([19, 24]). A topological space $X$ is called a semilocally $P$-space if for each $x \in X$ and each open set $U$ containing $x$ there is an open set $V$ such that $x \in V \subseteq U$ and $X-V$ is the union of finitely many closed $P$-sets.

Definition 6.2. A topological space $X$ is said to be $P$-Hausdorff if any two distinct points are contained in disjoint open sets having $P$-complements.

Clearly, every $P$-Hausdorff space is Hausdorff and the converse is not true in general. The table illustrates the type of semilocally $P$-space and $P$-Hausdorf space determined by a property $P$ and also reflects upon the relationship between the two notions.

Proposition 6.1. If $P$ denotes a finitely additive property, then every Hausdorff semilocally $P$-space is $P$-Hausdorff.

ProOf: Let $X$ be a Hausdorf semilocally $P$-space and let $x, y$ be any pair of distinct points in $X$. Then there exist disjoint open sets $U$ and $V$ containing $x$ and $y$, respectively. Since $X$ is also a semilocally $P$-space, there are disjoint open sets $U_{1}$ and $V_{1}$ such that

$$
x \in U_{1} \subseteq U \text { and } y \in V_{1} \subseteq V
$$


and both $X-U_{1}$ and $X-V_{1}$ are finite unions of closed $P$-sets. Since $P$ is finitely additive, $X-U_{1}$ and $X-V_{1}$ are $P$-sets and so $X$ is $P$-Hausdorff.

TheORem 6.2. If $f, g: X \rightarrow Y$ are $P$-continuous functions into a $P$-Hausdorff space $Y$, then the equaliser $E=\{x \in X \mid f(x)=g(x)\}$ of $f$ and $g$ is a closed subset of $X$. In particular, a $P$-continuous retract of a $P$-Hausdorff space is closed.

Proof: We shall show that $X-E$ is open. To this end, let $x \in X-E$. Then $f(x) \neq g(x)$ and so by the hypothesis on $Y$, there exist disjoint open sets $U$ and $V$ containing $f(x)$ and $g(x)$, respectively, such that $Y-U$ and $Y-V$ are $P$-sets. Since $f$ and $g$ are $P$-continuous, in view of Theorem 3.1 it follows that $f^{-1}(U)$ and $g^{-1}(V)$ are open sets in $X$ containing $x$. Let $G=f^{-1}(U) \cap g^{-1}(V)$. Then $G$ is an open set containing $x$ and disjoint from $E$ and so $E$ is closed in $X$.

The last part of the theorem now follows by taking $f$ to be the identity mapping on $X$ and $g$ to be the retraction mapping.

Corollary 6.3. The set of fixed points of a $P$-continuous function into a $P$ Hausdorff space is closed.

Corollary 6.4. If two $P$-continuous functions into a $P$-Hausdorff space coincide on a dense subset of the domain, then they are identical.

REMARK 6.1. According to the table, Theorem 6.2 contains several results in the literature. For example, with $P=$ regularly closed it shows that the equaliser of two almost continuous functions into a Hausdorf space is closed, a result due to Long and Herrington [32, Theorem 4] (also Noiri [39, Theorem 7]) and hence an almost continuous retract of a Hausdorff space is closed (Long and Carnahan [29, Theorem 3]). Similarly, with $P=$ zero set it yields that the equaliser of two $z$-continuous functions into a functionally Hausdorff space is closed and hence every $z$-continuous retract of a functionally Hausdorff space is closed.

Theorem 6.5. Let $f: X \rightarrow Y$ be a $P$-continuous function from a space $X$ into a $P$-Hausdorff space $Y$. Then the set $\left\{\left(x_{1}, x_{2}\right) \mid f\left(x_{1}\right)=f\left(x_{2}\right)\right\}$ is closed in the product space $X \times X$.

Proof: Let $A=\left\{\left(x_{1}, x_{2}\right) \mid f\left(x_{1}\right)=f\left(x_{2}\right)\right\}$. To show that $A$ is closed, let $(x, z) \notin A$. Then $f(x) \neq f(z)$. Since $Y$ is $P$-Hausdorff, there are disjoint open sets $U$ and $V$ containing $f(x)$ and $f(z)$, respectively and having $P$-complements. In view of the $P$-continuity of $f$, it follows that $f^{-1}(U)$ and $f^{-1}(V)$ are open sets in $X$ containing $x$ and $z$, respectively, and so $f^{-1}(U) \times f^{-1}(V)$ is an open set containing $(x, z)$ disjoint from $A$. Hence $A$ is closed in $X \times X$.

REMARK 6.2. If $P$ denotes regularly closed set, then the above theorem yields Theorem 8 of Noiri [39] pertaining to almost continuous functions. 


\begin{tabular}{|c|c|c|}
\hline S.No. & Property $P$ & $\begin{array}{l}\text { Type of } P \text {-continuous } \\
\text { function }\end{array}$ \\
\hline 1. & closed & continuous \\
\hline 2. & regularly closed & almost continuous $[\mathbf{5 1}]$ \\
\hline 3. & $\delta$-closed & almost continuous \\
\hline 4. & $\pi$-set & almost continuous \\
\hline 5. & finite set & $\begin{array}{l}\text { inverses of finite } \\
\text { closed } \\
\text { sets are closed }\end{array}$ \\
\hline 6. & countable & $\begin{array}{l}\text { inverses of countable } \\
\text { closed sets are closed }\end{array}$ \\
\hline 7. & cardinality $\leq m$ & $\begin{array}{l}\text { inverses of closed } \\
\text { sets of cardinality } \\
\leq m \text { are closed }\end{array}$ \\
\hline 8. & compactness & $c$-continuous $[12]$ \\
\hline 9. & countable compactness & $c^{*}$-continuous $[46]$ \\
\hline 10. & quasi $H$-closed & $H$-continuous $[30]$ \\
\hline 11. & Lindelöf property & $\ell$-continuous $[23]$ \\
\hline 12. & $N$-closed & $N$-continuous $[36]$ \\
\hline 13. & clopen set & mildly continuous $[54]$ \\
\hline 14. & connected set & $s$-continuous $[20]$ \\
\hline
\end{tabular}




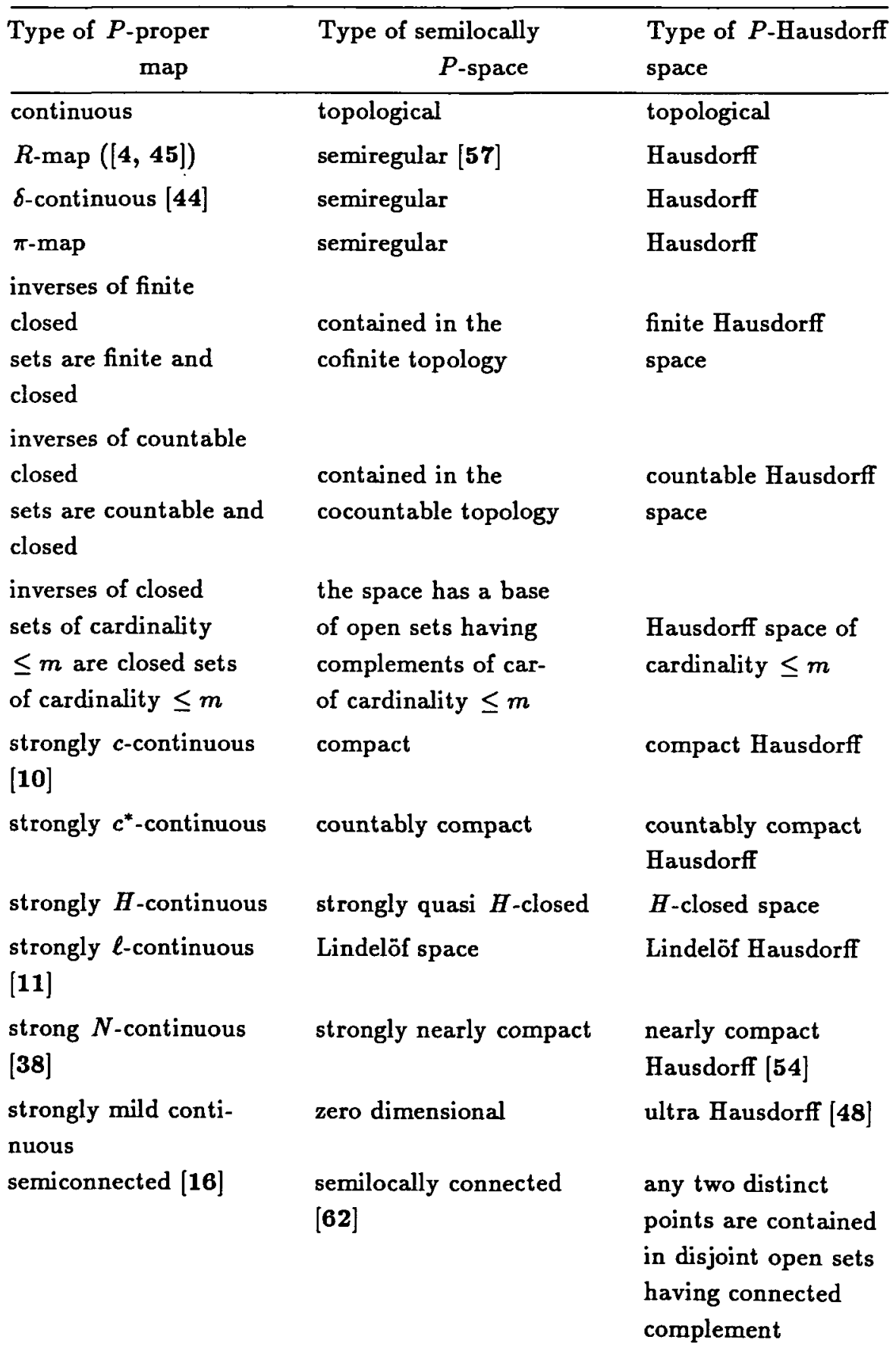




\begin{tabular}{lll}
\hline S.No. & Property $P$ & $\begin{array}{l}\text { Type of } P \text {-continuous } \\
\text { function }\end{array}$ \\
\hline 15. & $\begin{array}{l}\text { having finitely many } \\
\text { components }\end{array}$ & $s$-continuous \\
\end{tabular}

16.
(a) zero set
(b) z-closed set

$z$-continuous [53]

$17^{+}$. being a ray of the

lower semicontinuous

type $\{x: x \leq a\}$

$18^{+}$. being a ray of the

upper semicontinuous

type $\{x: x \geq a\}$

$19^{*}$. having increasing

complement

20. paracompactness

$\tau_{i}$-continuous $[\mathbf{1}]$

21. regularly closed

para-continuous [35]

and compact

almost $c$-continuous

[58]

22. quasi $\boldsymbol{H}$-closed

almost $H$-continuous [9]

regularly closed

set

23. being the boundary

weak $^{*}$ continuous $[\mathbf{2 7}][50]$

of an open set

24. $G_{\delta \text {-set }}$

$D$-continuous [25]

25. strongly closed

$G_{\delta}$

26. point closure

weakly $D$-continuous [26]

inverse images of point

closures are closed

$27^{++} . \quad$ hard set $[49]$

$h$-continuous [26]

28. strongly regularly

rather weak almost

closed

continuous [59]

+ Here the underlying space is either the real line with the usual topology

* Here the underlying space is a partially ordered topological space.

+ Here the space is assumed to be a Tychonoff space. 


\begin{tabular}{|c|c|c|}
\hline $\begin{array}{c}\text { Type of } P \text {-proper } \\
\text { map }\end{array}$ & $\begin{array}{r}\text { Type of semilocally } \\
P \text {-space }\end{array}$ & $\begin{array}{l}\text { Type of } P \text {-Hausdorff } \\
\text { space }\end{array}$ \\
\hline nearly semiconnected & semilocally connected & $\begin{array}{l}\text { any two distinct points } \\
\text { are contained in disjoint } \\
\text { open sets having } \\
\text { finitely many components }\end{array}$ \\
\hline$z$-continuous & completely regular & functionally Hausdorff \\
\hline- & $\begin{array}{l}\text { right order topology } \\
\text { [56] }\end{array}$ & - \\
\hline- & left order topology [56] & - \\
\hline - & $\begin{array}{l}\text { the space has a base of } \\
\text { increasing open sets }[1]\end{array}$ & - \\
\hline- & paracompact & paracompact Hausdorff \\
\hline- & semiregular compact & compact Hausdorff \\
\hline- & $\begin{array}{l}\text { semiregular quasi } \\
H \text {-closed }\end{array}$ & minimal Hausdorff \\
\hline - & - & - \\
\hline strongly $D$-continuous & $D$-regular $[14]$ & $D$-Hausdorff [25] \\
\hline weakly $D$-continuous & $\begin{array}{l}D \text {-complelely regular } \\
{[2]}\end{array}$ & $\begin{array}{l}\text { strongly } D \text {-Hausdorff } \\
{[26]}\end{array}$ \\
\hline $\begin{array}{l}\text { inverse images of } \\
\text { point closures are } \\
\text { point closures }\end{array}$ & $\begin{array}{l}\text { the space has a base of } \\
\text { open sets which are } \\
\text { complements of closures } \\
\text { of finite sets }\end{array}$ & $\begin{array}{l}\text { at most a two point } \\
\text { discrete space }\end{array}$ \\
\hline$h$-proper map [26] & $\begin{array}{l}\text { realcompact space }[15] \\
\text { strongly semiregular } \\
\text { space }[59]\end{array}$ & $\begin{array}{l}\text { realcompact space } \\
\text { Hausdorff }\end{array}$ \\
\hline
\end{tabular}

or a linearly order space endowed with the order topology. 


\section{REFERENCES}

[1] Gerald Beer, 'Lattice semicontinuous mappings and their applications', Houston J. Math. 13 (1987), 303-318.

[2] H. Brandenburg, 'On a class of nearness spaces and the epireflective hull of developable topological space', Proc. Internat. Topological Symp. (Belgrade 1977).

[3] H. Brandenburg, 'On spaces with a $G_{\delta}$-basis', Arch. Math. 35 (1980), 544-547.

[4] D. Carnahan, Some properties related to compactness in topological spaces (Ph.D Thesis, Univ. of Arkansas, 1973).

[5] D. Carnahan, 'Locally nearly compact spaces', Boll. Un. Mat. Ital. 6 (1972), 146-153.

[6] F. Cammaroto and G. Lo Faro, 'Sulle funzioni $\gamma$-continue', Le Matematiche 35 (1980), 1-17.

[7] F. Commaroto and T. Noiri, 'On WC-continuous functions', J. Korean Math. Soc. 24 (1987), 11-19.

[8] S.P. Franklin, 'Spaces in which sequences suffice', Fund. Math. 57 (1965), 107-115.

[9] D.B. Gauld, 'Topologies related to notions of near continuity', Kyungpook Math. J. 21 (1981), 195-204.

[10] D.B. Gauld, M. Mrsevic, I.L. Reilly and M. K. Vamanamurthy, 'Continuity properties of functions', Colloquia Math. Soc. Janos Bolyai 41. : Topology and its Applications, Eger (1983), pp. 311-322.

[11] D.B. Gauld, M. Mrsevic, I.L. Reilly and M. K. Vamanamurthy, 'Colindlöf topologies and

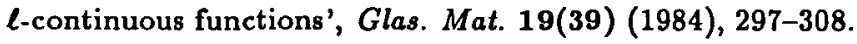

[12] Karl R. Gentry and Hughes B. Hoyle, 'c-continuous functions', Yokohama Math. J. 18 (1970), 71-76.

[13] N.C. Heldermann, 'The category of $D$-completely regular spaces is simple', Trans. Amer. Math. Soc. 262 (1980), 437-446.

[14] N.C. Heldermann, 'Developability and some new regularity axioms', Canad. J. Math. 33 (1981), 641-663.

[15] E. Hewitt, 'Rings of real-valued continuous functions I', Trans. Amer. Math. Soc. 64 (1948), 45-99.

[16] John Jones, Jr., 'On semiconnected mappings of topological spaces', Proc. Amer. Math. Soc. 19 (1968), 174-175.

[17] Victor Klee, 'Stability of the fixed point property', Colloq. Math. 8 (1961), 43-46.

[18] Victor Klee and Andre Yandl, 'Some proximate concepts in topology', Sympos. Math. 16 (1975), 21-39.

[19] J.K. Kohli, 'A unified approach to continuous and certain non-continuous functions', Symposium on Gen. Topology and its Applications, University of Delhi, Delhi (1978). J. Austra. Math. Soc. (to appear).

[20] J.K. Kohli, 'A class of mappings containing all continuous and all semiconnected mappings', Proc. Amer. Math. Soc. 72 (1978), 175-181.

[21] J.K. Kohli, ' $S$-continuous functions, certain weak forms of regularity and complete regularity', Math. Nachr 97 (1980), 189-196. 
[22] J.K. Kohli, ' $S$-continuous functions, certain weak forms of normality and strongly semilocally connected spaces', Math. Nachr 99 (1980), 69-76.

[23] J.K. Kohli, 'A class of mappings containing all continuous mappings', Glas. Mat. 16(36) (1981), 361-368.

[24] J.K. Kohli, 'A unified view of (complete) regularity and certain variants of (complete) regularity', Canad. J. Math. 36 (1984), 783-794.

[25] J.K. Kohli, ' $D$-continuous functions, $D$-Hausdorf spaces and $D$-regular spaces', (submitted).

[26] J.K. Kohli, 'On certain generalized notions of continuity', (preprint).

[27] Norman Levine, 'A decomposition of continuity in topological spaces', Amer. Math. Monthly 68 (1961), 44-46.

[28] Paul E. Long, 'Concerning semiconnected mappings', Proc. Amer. Math. Soc. 21 (1969), 117-118.

[29] Paul E. Long and D. A. Carnahan, 'Comparing almost continuous functions', Proc. Amer. Math. Soc. 38 (1973), 413-418.

[30] Paul E. Long and T.R. Hamlett, ' $H$-continuous functions', Boll. Un. Mat. Ital 11 (1975), 552-558.

[31] Paul E. Long and Michael D. Hendrix, 'Properties of c-continuous functions', Yokohama Math. J. 22 (1974), 117-125.

[32] Paul E. Long and Larry L. Herrington, 'Properties of almost continuous functions', Boll. Un. Mat. Ital 10 (1974), 336-342.

[33] Paul E. Long and Larry L. Herrington, 'Properties of $c$-continuous and $c^{*}$-continuous functions', Kyungpook Math. J. 15 (1975), 213-221.

[34] Paul E. Long and Larry L. Herrington, 'Functions with strongly closed graphs', Boll. Un. Mat. Ital 12 (1975), 381-384.

[35] Paul E. Long and Larry L. Herrington, 'Para-continuous functions', Egypt, Proc. Math. Phys. Soc. 52 (1985), 1-5.

[36] S.R. Malghan and V.V. Hanchinamani, ' $N$-continuous functions', Bruxelles, Ann. Soc. Sci. 88 (1984), 69-79.

[37] Asha Mathur, ' $\delta$-continuous mappings', (preprint).

[38] M. Mrsevic and I.L. Reilly, 'On $N$-continuity and co $N$-closed topologies', Recerche di Matematica (to appear).

[39] Takashi Noiri, 'Between continuity and weak continuity', Boll. Un. Mat. Ital. 9 (1974), 647-654.

[40] Takashi Noiri, 'A remark on almost continuous mappings', Proc. Japan Acad. 50 (1974), 205-207.

[41] Takashi Noiri, 'On functions with strongly closed graphs', Acta Math. Hungar. 32 (1978), $1-4$.

[42] Takashi Noiri, 'Properties of $H$-continuous functions', Res. Rep. of Yatsushiro National College of Technology 1 (1979), 85-90.

[43] Takashi Noiri, 'Properties of almost c-continuous functions', J. Korean Math. Soc. 15 
(1979), 85-90.

[44] Takashi Noiri, ' $\delta$-continuous functions', J. Korean Math. Soc. 16 (1980), 161-166.

[45] Takashi Noiri, 'Strong forms of continuity in topological spaces', Rend. Circ. Mat. Palermo (2) Suppl. 12 (1986), 107-113.

[46] Yang Su Park, 'c '-continuous functions', J. Korean Math. Soc. 8 (1971), 69-72.

[47] J.R. Porter and J. Thomas, 'On $H$-closed and minimal Hausdorff spaces', Trans. Amer. Math. Soc. 18 (1969), 159-170.

[48] J.R. Porter and R.G. Woods, 'Ultra-Hausdorff H-closed extensions', Pacific J. Math. 84 (1979), 399-411.

[49] Marlon C. Rayburn, 'On hard sets', Gen. Topol. Appl. 6 (1976), 21-26.

[50] D.A. Rose, 'On Levine's decomposition of continuity', Canad. Math. Bull. 21 (1978), 477-481.

[51] M.K. Singal and A.R. Singal, 'Almost continuous functions', Yokohama Math. J. 16 (1968), 63-73.

[52] M.K. Singal and Asha Mathur, 'Nearly compact spaces', Boll. Un. Mat. Ital 2 (1969), 702-710.

[53] M.K. Singal and S.B. Niemse, ' $z$-continuous functions', Yokohama Math. J.

[54] M.K. Singal and R.C. Jain, 'Mildly continuous functions', (preprint).

[55] I.J. Singh and R. Prasad, 'Almost c-continuous functions', Indian J. Math. 27 (1985), 165-168.

[56] L.A. Steen and J.A. Seebach Jr., Counterexamples in topology (Springer-Verlag, Berlin, Heidelberg, New York, 1978).

[57] M.H. Stone, 'Applications of the theory of Boolean rings to general topology', Trans. Amer. Math. Soc. 41 (1937), 375-481.

[58] G.H. Suk, 'Almost c-continuous functions', J. Korean Math. Soc. 14 (1978), 229-234.

[59] J. Tong, 'Weak almost continuous mappings and weak nearly compact spaces', Bell. Un. Mat. Ital (6) 1-A (1982), 385-381.

[60] N.V. Veličko, 'On $H$-closed spaces', (Russian), Mat. Sb. 70 (1966), 98-112. translated as, Amer. Math. Soc. Transl. 78 (1968), 103-118.

[61] D.W. Wagner, 'Semi-compactness with respect to Euclidean cone', Canad. J. Math. 29 (1977), 29-36.

[62] G.T. Whyburn, 'Semilocally connected sets', Amer. J. Math. 61 (1939), 733-741.

[63] V. Zaičev, 'Some classes of topological spaces and their bicompactifications', Soviet Math. Dokl. 9 (1968), 192-193.

Department of Mathematics

Hindu College

University of Delhi

Delhi - 110007

India 